九州大学学術情報リポジトリ

Kyushu University Institutional Repository

\title{
ON ALGORITHMS TO OBTAIN DIGRAPHS REPRESENTING DYNAMICAL BEHAVIORS ASSOCIATED WITH A SINGLE NEURONIC EQUATION
}

Tagawa, Shojiro

International Institute for Advanced Study of Social Information Science

https://doi.org/10.5109/13106

出版情報: 統計数理研究. 17 (1/2), pp. 85-91，1976-03. Research Association of Statistical Sciences

バージョン：

権利関係 : 


\title{
ON ALGORITHMS TO OBTAIN DIGRAPHS REPRESENTING DYNAMICAL BEHAVIORS ASSOCIATED WITH A SINGLE NEURONIC EQUATION
}

\author{
By
}

\author{
Shôjirô TAGAWA*
}

(Received June 30, 1975)

\section{Introduction.}

This paper is concerned with digraphs representing dynamical behaviors associated with a single neuronic equation with time lag $(n-1)$ which is introduced by Caianiello [1]. In order to investigate digraphs themselves and the transition phenomena of the digraphs, we shall present the algorithms to obtain the family of digraphs. Our situation is on the same spirit of Kitagawa's method [2]. Indeed, we first determine all possible order relations of $2^{n}$ terms corresponding to state configurations. Next, we obtain the family of digraphs by varying the threshold for each order relation. It is noticed that this family is compared with the family of simultaneously realizable threshold functions (see Muroga [6]) in threshold logic.

In Section 2, we shall summarize definitions and preliminary results indispensable in this paper, many of which are stated in [2]. In Section 3, we shall present an algorithm to obtain all possible order relations of $z^{(r)}$ 's concerning with $i_{0} i_{1} \cdots i_{n-1}$ in terms of notations in [2] on the basis of all possible order relations corresponding to $i_{0} i_{1} \cdots i_{n-2}$. The problem to determine the above order relation is essentially equivalent to the problem to check the consistency of the system of inequalities. In particular, the problem to determine order relation of $z^{(r)}$ and $z^{(s)}$ under the condition that some order relation of $z^{(t)}$ 's is given, where $z^{(s)}$ is contained in the set of $z^{(r)}$ 's but $z^{(t)}$ is not, is separately discussed in Section 4 . In Section 5 , we shall present an algorithm to obtain the family of digraphs by varying the threshold in each order relation given in Section 4. There are some concepts similar to our arguments, for example, variable-threshold threshold elements (see [5]), simultaneously realizable threshold functions and lattice of canonical positive threshold functions (see [6]) in threshold logic. We shall discuss the relations among those notions and our arguments.

\section{Definitions and preliminaries.}

We shall consider a single neuronic equation with time lag $(n-1)$ :

$$
x(t+1)=1\left[\sum_{k=0}^{n-1} a_{k} x(t-k)-\theta\right]
$$

* International Institute for Advanced Study of Social Information Science, Fujitsu Limited, 17-25, Shinkamata 1-Chome, Ohta-ku, Tokyo, Japan. 
where the variables $x(t-k)$ and $x(t+1)$ assume 0 or 1 , the coefficients $a_{k}, k=0,1, \cdots$, $n-1$, and a threshold $\theta$ are real numbers and $1[u]=1$ if $u>0$ and $=0$ if $u \leqq 0$.

Let us set $a=\left(a_{0}, a_{1}, \cdots, a_{n-1}\right)$ and $\delta=\left(\delta_{0}, \delta_{1}, \cdots, \delta_{n-1}\right)$ where $\delta_{k}=x(t-k)$ for $k=0$, $1, \cdots, n-1$. Let $X_{n}$ denote the set of all possible $\delta$ 's. We now introduce the types of subdivisions of the coefficient space :

$$
\begin{aligned}
& i_{0} i_{1} \cdots i_{n-1} \equiv\left\{a \mid 0<a_{i_{0}}<a_{i_{1}}<\cdots<a_{i_{n-1}}\right\} \\
& \bar{i}_{0} i_{1} \cdots i_{n-1} \equiv\left\{a\left|a_{i_{0}}<0<\right| a_{i_{0}} \mid<a_{i_{1}}<\cdots<a_{i_{n-1}}\right\} \\
& i_{0} \bar{i}_{1} \cdots i_{n-1} \equiv\left\{a\left|a_{i_{1}}<0<a_{i_{0}}<\right| a_{i_{1}} \mid<\cdots<a_{i_{n-1}}\right\} \\
& \bar{i}_{0} \bar{i}_{1} \cdots i_{n-1} \equiv\left\{a\left|a_{i_{1}}<a_{i_{0}}<0<\right| a_{i_{0}}|<| a_{i_{1}} \mid<\cdots<a_{i_{n-1}}\right\} \\
& \quad \vdots \\
& \bar{i}_{0} \bar{i}_{1} \cdots \bar{i}_{n-1} \equiv\left\{a \mid a_{i_{n-1}}<a_{i_{n-2}}<\cdots<a_{i_{1}}<a_{i_{0}}<0\right\},
\end{aligned}
$$

where $i_{k}$ runs over the set $\{0,1, \cdots, n-1\}$.

Let us define

$$
i_{k}^{\varepsilon}=\left\{\begin{array}{lll}
i_{k} & \text { if } & \varepsilon=1, \\
\overline{i_{k}} & \text { if } & \varepsilon=0,
\end{array}\right.
$$

and

$$
z^{(r)} \equiv \sum_{k=0}^{n=1} a_{k} \delta_{k}^{(r)} \quad \text { for all } \quad \delta^{(r)} \in X_{n}
$$

Then, each subdivision of $(2.2)$ is rewritten as $i_{0} \varepsilon_{0} i_{1}^{\varepsilon_{1}} \ldots i_{n-1}^{\varepsilon_{n-1}}$.

As stated in [2], for $n \geqq 3$ there are more than one types of the ascending orders of the values of $z^{(r)}$ 's corresponding to the subdivisions of the coefficient space. For example, let $n=3$, then

$$
i_{0} i_{1} i_{2} \rightleftarrows 0<a_{i_{0}}<a_{i_{1}}<\left[\begin{array}{c}
a_{i_{0}}+a_{i_{1}} \\
a_{i_{2}}
\end{array}\right]<a_{i_{0}}+a_{i_{2}}<a_{i_{1}}+a_{i_{2}}<a_{i_{0}}+a_{i_{1}}+a_{i_{2}},
$$

so that there are two types of the ascending orders of $\left\{z^{(1)}, \cdots, z^{(8)}\right\}$. Note that each $i_{0} \varepsilon_{0} i_{1}{ }_{1} i_{2}{ }^{\varepsilon_{2}}$ has just as the same types of the ascending orders as those in $i_{0} i_{1} i_{2}$. Consequently, we can confine ourselves only to the types corresponding to $i_{0} i_{1} \cdots i_{n-1}$.

The set $X_{n}$ is called to be arrayed in an inverse lexical order if it is enumerated as follows :

$\begin{array}{ccccccccccccc}(1) & (2) & (3) & (4) & (5) & (6) & (7) & (8) & \cdots \cdots & \left(2^{n}-3\right) & \left(2^{n}-2\right) & \left(2^{n}-1\right) & \left(2^{n}\right) \\ 0 & 1 & 0 & 1 & 0 & 1 & 0 & 1 & \cdots \cdots & 0 & 1 & 0 & 1 \\ 0 & 0 & 1 & 1 & 0 & 0 & 1 & 1 & \cdots \cdots & 0 & 0 & 1 & 1 \\ 0 & 0 & 0 & 0 & 1 & 1 & 1 & 1 & \cdots \cdots & 1 & 1 & 1 & 1 \\ \vdots & \vdots & \vdots & \vdots & \vdots & \vdots & \vdots & \vdots & \cdots \cdots & \vdots & \vdots & \vdots & \vdots \\ 0 & 0 & 0 & 0 & 0 & 0 & 0 & 0 & \cdots \cdots & 1 & 1 & 1 & 1\end{array}$

and each vector in $X_{n}$ is numbered as above. 


\section{An algorithm to obtain all possible types of the ascending orders of $\left\{z^{(1)}, \cdots, z^{\left(2^{n}\right)}\right\}$ corresponding to $i_{0} i_{1} \cdots i_{n-1}$.}

We now present the algorithm which generates all possible types of the ascending orders of $z^{(r)}$ 's corresponding to $i_{0} i_{1} \cdots i_{n}$ when the all possible types corresponding to $i_{0} i_{1} \cdots i_{n-1}$ are given, i. e., this algorithm constructs all possible types of $z^{(r)}$ 's in $(n+1)$ dimensional coefficient space from those in $n$-dimensional coefficient space.

Assume that one orderrelation corresponding to $i_{0} i_{1} \ldots i_{n-1}$ :

$$
z^{(1)}<z^{(2)}<\cdots<z^{(2 n)}
$$

is given.

Step 1: Let $k=2$.

Step 2: Add a new number $a_{n}$ to every $z^{(r)}$ and define

$$
z^{\left(2^{n}+r\right)} \equiv z^{(r)}+a_{n} \quad \text { for } \quad r=1,2, \cdots, 2^{n} .
$$

It is then clear that

$$
z^{\left(2^{n+1)}\right.}<z^{\left(2^{n}+2\right)}<\cdots<z^{\left(2^{n+1}\right)} .
$$

Step 3: Compare the value of $z^{\left(2^{n}+1\right)}$ with each of $z^{(1)}, \cdots, z^{\left(2^{n}\right)}$, and enumerate all possible order relations of numbers $z^{(1)}, \cdots, z^{\left(2^{n}\right)}$ and $z^{\left(2^{n}+1\right)}$.

Step 4: On the basis of order relations given in Step 3, compare $z^{\left(2^{n}+k\right)}$ with $z^{(r)}, r=1, \cdots, 2^{n}+k-1$, and determine all possible order relations of numbers $z^{(r)}, r=1,2, \cdots, 2^{n}+k$.

Step 5: Execute Step 4 with $k$ replaced by $k+1$, and repeat until $k=2^{n}$.

Note that $z^{(1)}=0, z^{(2)}=a_{i_{0}}$, and $z^{(3)}=a_{i_{1}}$ whenever we are concerned with $i_{0} i_{1} \cdots i_{n-1}$. Moreover, it suffices to consider only the case that $012 \cdots(n-1)$, i. e., $i_{j}=j, j=0,1$, $\cdots, n-1$.

Let us number all vectors $\delta^{(r)}$ in $X_{n}$ in an inverse lexical order as in (2.3). Then, we give the number $r$ to $z^{(r)}=\sum_{k=0}^{n-1} a_{k} \delta_{k}^{(r)}$ for $1 \leqq r \leqq 2^{n}$. Now, the above algorithm turns out to be as follows:

Assume that the sequence of positive integers $j_{1} j_{2} \cdots j_{2}$ where $1 \leqq j_{r} \leqq 2^{n}, r=1, \cdots, 2^{n}$, which corresponds to an order relation of $\left\{z^{(1)}, \cdots, z^{\left(2^{n}\right)}\right\}$.

Step 1: Set $p=1$.

Step 2: Generate numbers $j_{r}, r=2^{n}+1, \cdots, 2^{n+1}$, as $j_{2^{n+r}}=j_{r}+2^{n}$ for $r=1, \cdots, 2^{n}$.

Step 3: Since $j=1$, in any case, we have $j_{2^{n+1}}=2^{n}+1$, and construct all the sequences with the number $j_{2^{n+1}}=2^{n}+1$ in addition to the given sequence. They are all sequences in which the number $2^{n}+1$ is located at the right of the number $2^{n-1}+1$.

Step $4:$ If the sequences $\left\{j_{r}\right\}_{r=1, \cdots, 2^{2}+p}, 1 \leqq p \leqq 2^{n}-1$, are given, then construct all possible $\left\{j_{r}\right\}_{r=1, \cdots, 2^{n}+p+1}$ by checking the location of $j_{2^{n}+p+1}$. This step is discussed in Section 4 in detail.

Step 5: Execute Step 4 with $p$ replaced by $p+1$ and repeat it until all possible sequences $\left\{j_{r}\right\}_{r=1, \cdots, 2^{n+1}}$ are obtained.

EXAMPLE. Let $n=3$, and suppose that the sequence 
is given.

Step 1: Construct the sequence by adding 8 to each number and we have

$$
\begin{array}{llllllll}
9 & 10 & 11 & 13 & 12 & 14 & 15 & 16 .
\end{array}
$$

Step 2: Determine all possible locations of the number 9 :

$\begin{array}{lllllllll}1 & 2 & 3 & 5 & 4 & 6 & 7 & 8 & 9 \\ 1 & 2 & 3 & 5 & 4 & 6 & 7 & 9 & 8 \\ 1 & 2 & 3 & 5 & 4 & 6 & 9 & 7 & 8 \\ 1 & 2 & 3 & 5 & 4 & 9 & 6 & 7 & 8 \\ 1 & 2 & 3 & 5 & 9 & 4 & 6 & 7 & 8 .\end{array}$

Step 3: Determine all possible locations of the number 10:

$\begin{array}{rrrrrrllrr}1 & 2 & 3 & 5 & 4 & 6 & 7 & 8 & 9 & 10 \\ 1 & 2 & 3 & 5 & 4 & 6 & 7 & 9 & 8 & 10 \\ 1 & 2 & 3 & 5 & 4 & 6 & 9 & 10 & 7 & 8 \\ 1 & 2 & 3 & 5 & 4 & 9 & 6 & 7 & 10 & 8 \\ 1 & 2 & 3 & 5 & 4 & 9 & 6 & 10 & 7 & 8 \\ 1 & 2 & 3 & 5 & 9 & 4 & 6 & 7 & 10 & 8 \\ 1 & 2 & 3 & 5 & 9 & 4 & 6 & 10 & 7 & 8 .\end{array}$

Step 4-9: Determine all possible locations of the number $j_{r+1}$ when all possible sequences of numbers $j_{1}, j_{2}, \cdots, j_{r}$ are given, where $j_{r+1}$ is selected in the order of $11,13,12,14,15$ and 16.

In the last step, we can obtain eight sequences, i. e.,

$\begin{array}{rrrrrrllrrrrrrrr}1 & 2 & 3 & 5 & 4 & 6 & 7 & 8 & 9 & 10 & 11 & 13 & 12 & 14 & 15 & 16 \\ 1 & 2 & 3 & 5 & 4 & 6 & 7 & 9 & 8 & 10 & 11 & 13 & 12 & 14 & 15 & 16 \\ 1 & 2 & 3 & 5 & 4 & 6 & 9 & 7 & 10 & 8 & 11 & 13 & 12 & 14 & 15 & 16 \\ 1 & 2 & 3 & 5 & 4 & 6 & 9 & 10 & 7 & 8 & 11 & 13 & 12 & 14 & 15 & 16 \\ 1 & 2 & 3 & 5 & 4 & 9 & 6 & 7 & 10 & 11 & 8 & 13 & 12 & 14 & 15 & 16 \\ 1 & 2 & 3 & 5 & 4 & 9 & 6 & 10 & 7 & 11 & 8 & 13 & 12 & 14 & 15 & 16 \\ 1 & 2 & 3 & 5 & 9 & 4 & 6 & 7 & 10 & 11 & 13 & 8 & 12 & 14 & 15 & 16 \\ 1 & 2 & 3 & 5 & 9 & 4 & 6 & 10 & 7 & 11 & 13 & 8 & 12 & 14 & 15 & 16 .\end{array}$

This algorithm was executed by the electronic computer F230-60 and we obtained 546 order relations for $n=5$ in one minute and thirty seconds. For $n=6$, it is not a good method to enumerate all possible order relations since there will be more than two hundred thousand ones.

\section{An algorithm to determine all possible locations of $j_{2^{n}+p+1}$.}

Given an order relation

$$
z^{(1)}<z^{(2)}<\cdots<z^{\left(2^{n}+p\right)},
$$

we need to know that the value $z^{\left(2^{n}+p+1\right)}$ is greater or less than some $z^{(r)}, 1 \leqq r \leqq 2^{n}+p$. 
This problem is equivalent to the following problem: Determine whether the system of inequalities

$$
z^{(1)}<z^{(2)}<\cdots<z^{(r)}<z^{\left(2^{n}+p+1\right)}<z^{(r+1)}<\cdots<z^{\left(2^{n}+p\right)}
$$

is consistent or not, for given $r, 1 \leqq r \leqq 2^{n}+p$.

For the problem of this type, there are known some methods such as (i) simplex method, (ii) Fourier-Motskin elimination method. But, since the system of inequalities is of the special form, we adopt the different and very simple method.

Assume that the sequence of positive integers $j_{1} j_{2} \cdots j_{2^{n}+p}$ is given as in Section 3.

Step 1: Set $k=0$ and $u=2^{n}+p+1$.

Step 2: Compare the number $j_{2^{n}+p+1}$ with $j_{2^{n}+p-k}$, and construct the vectors $\delta^{\left(j_{2}{ }^{n}+p-k\right)}$ and $\delta^{\left(j_{2} n+p+1\right)}$ corresponding to $j_{2^{n}+p-k}$ and $j_{2^{n}+p+1}$, respectively (see (2.3)). If $i$-th components of two vectors $\delta^{\left(j_{2} n^{n}+p-k\right)}$ and $\delta^{\left(j_{2} n+p+1\right)}$ have the same value 1 , then replace those components by 0 for $i, 1 \leqq i \leqq n$. These two vectors are denoted by $\tilde{\delta}^{\left(j_{2} n+p-k\right)}$ and $\tilde{\delta}^{\left(j_{2}{ }^{n}+p+1\right)}$, respectively.

Step 3: Find the two numbers $l_{2 n_{+p-k}}$ and $l_{2 n_{+p+1}}$ corresponding to two vectors $\tilde{\delta}^{\left(j_{2} n+p-k\right)}$ and $\tilde{\delta}^{\left(j_{2} n+p+1\right)}$, respectively, in (2.3).

Step 4: Search the sequence $j_{1} j_{2} \cdots j_{2^{n}+p}$ and determine which case (a) $\cdots l_{2 n+p-k}$ $\cdots l_{2^{n}+p+1} \cdots$ or (b) $\cdots l_{2^{n}+p+1} \cdots l_{2^{n}+p-k} \cdots$ arises. If the case (a) arises, then go to Step 6. If the case (b) arises, then go to Step 5. If neither (a) nor (b) arises, then go to Step 2 with $k$ replaced by $k+1$.

Step 5: Let $n=2^{n}+p-k$ and go to Step 2 with $k$ replaced by $k+1$.

Step 6: Construct all sequences in which the number $j_{2^{n}+p+1}$ is placed between $j_{2^{n+p-k}}$ and $j_{u}$. Consequently, we can obtain

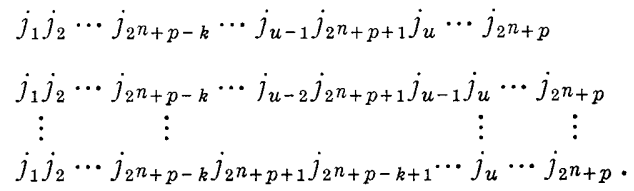

It is easy to verify that the system of inequalities corresponding to the sequences obtained by the above algorithm is necessarily consistent and that all possible order relations are exhausted.

\section{An algorithm to obtain digraphs associated with a single neuronic equation.}

The digraphs associated with a single neuronic equation (2.1) are derived as follows (see [2] for the detail description): Define the operator $L$ from $X_{n}$ into $X_{n}$ by

$$
L\left(\begin{array}{c}
\delta_{0} \\
\delta_{1} \\
\vdots \\
\delta_{n-1}
\end{array}\right)=\left(\begin{array}{c}
1\left[\sum_{k=0}^{n-1} a_{k} \delta_{k}-\theta\right] \\
\delta_{0} \\
\vdots \\
\delta_{n-2}
\end{array}\right),
$$

and we write 


$$
\begin{gathered}
\left(\begin{array}{c}
\delta_{0} \\
\delta_{1} \\
\vdots \\
\delta_{n-1}
\end{array}\right) \rightarrow L\left(\begin{array}{c}
\delta_{0} \\
\delta_{1} \\
\vdots \\
\delta_{n-1}
\end{array}\right)=\left(\begin{array}{c}
1 \\
\delta_{0} \\
\vdots \\
\delta_{n-2}
\end{array}\right) \quad \text { if } 1\left[\sum a_{k} \delta_{k}-\theta\right]=1 \\
\left(\begin{array}{c}
\delta_{0} \\
\delta_{1} \\
\vdots \\
\delta_{n-1}
\end{array}\right) \rightarrow L\left(\begin{array}{c}
\delta_{0} \\
\delta_{1} \\
\vdots \\
\delta_{n-1}
\end{array}\right)=\left(\begin{array}{c}
0 \\
\delta_{0} \\
\vdots \\
\delta_{n-2}
\end{array}\right) \quad \text { if } 1\left[\sum a_{k} \delta_{k}-\theta\right]=0 .
\end{gathered}
$$

We number all state configurations in $X_{n}$ in an inverse lexical order as (2.3). For $n=3$, we draw the digraphs as Fig. 1 . For $n=4$, we have Fig. 2.

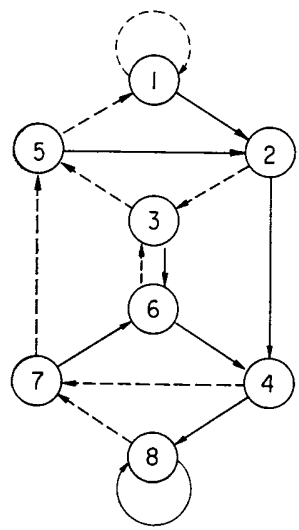

Fig. 1 .

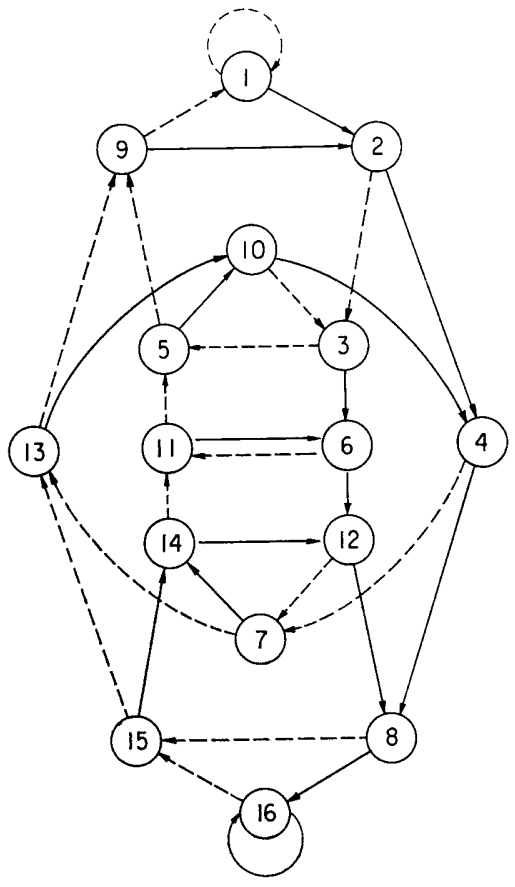

Fig. 2.

We shall investigate how digraphs change as $\theta$ varies. It is then sufficient that we make $\theta$ vary for each order relation obtained by the algorithm introduced in Section 3 .

Assume that the sequence $j_{1} j_{2} \cdots j_{2^{n}}$ is given.

Step 1: Let $k=0$.

Step 2: Classify the sequence into two subsets $\left\{j_{1} j_{2} \cdots j_{k}\right\}\left\{j_{k+1} \cdots j_{2}\right.$ \} which correspond to the set of $z^{(r)}$ less than the threshold $\theta$ and the set of $z^{(r)}$ greater than $\theta$, respectively.

Step 3: Draw the digraph in which the operator corresponding to the number in $\left\{j_{1} j_{2} \cdots j_{k}\right\}$ is of the type (5.2) and the operator corresponding to the number in $\left\{j_{k+1} \cdots j_{2^{n}}\right\}$ is of the type (5.1). Go to Step 3 with $k$ replaced by $k+1$. 


\section{Discussions.}

There are several arguments in threshold logic similar to those described in the preceding sections. For example, there are theories of variable-threshold threshold elements, see [5], and the lattice of canonical positive functions $L_{n}{ }^{(2)}$ in Muroga [6]. We shall compare these concepts with our arguments.

If we introduce the ordering relation $\gtrsim$ stated in $[6, p .113]$ and the canonical functions, then the fact that we confine ourselves to the subdivision $i_{0} i_{1} \cdots i_{n-1}$ corresponds to the fact that only the canonical positive functions come into question. The lattice $L_{n}{ }^{(2)}$ given in $[6$, p. 237] is compared with the set of digraphs obtained by Section 5 from the one sequence $j_{1} j_{2} \cdots j_{2} n$. The number of lattices in $L_{n}{ }^{(2)}$ with $x_{n} \succsim x_{n-1} \succsim \cdots \succsim x_{1}$, corresponds to the number of all possible sequences $j_{1} j_{2} \cdots j_{2}$.

In comparison with the arguments of Meisel [5], the set of digraphs obtained in Section 5 corresponds to the case where " $S$-partition introduced by $F$ " [5, p. 661] is $S=\left\{S_{1}, S_{2}, \cdots, S_{2^{n}}\right\}$ and each $S_{r}, 1 \leqq r \leqq 2^{n}$, consists of a single element. Furthermore, the backtrack programming stated in $[5$, p. 665$]$ is contrasted with the algorithm introduced in Section 4.

Acknowledgment. The author is indebted to Director Tosio Kitagawa for his suggestions and helpful discussions.

\section{References}

[1] E.R. CaiAniello, Outline of a theory of thought processes and thinking machines, J. Theoret. Biol. I (1961), pp. 204-235.

[2] T. KITAgawA, Dynamical behaviours associated with a system of $N$ neuroeic equations with time lag, (I), Research Institute of Fundamental Information Science, Kyushu Univ., Research Report No. 46, November, 1974.

[3] T. Kitagawa, Dynamical Behaviours associated with a system of $N$ neuronic equations with time lag, $(I I), N=1, n=4$, Research Institute of Fundamental Information Science, Kyushu Univ., Research Report No. 49, 1975.

[4] T. KITAgawa, Dynamical behaviours associated with a system of $N$ neuronic equations with time lag, $(I I I), N=1, n=5$, Research Institute of Fundamental Information Science, Kyushu Univ., Research Report No. 54, 1975.

[5] W.S. MEIsel, Variable-threshold threshold elements, IEEE Trans. Computer, Vol. C-17, pp. 656-667, July 1968.

[6] S. Muroga, Threshold Logic and its Applications, Wiley-Interscience, 1971.

[7] S. TAGAWA, Sufficient conditions for switching functions to be threshold ones and their applications, Bull. Math. Statist., 17 (1975), pp. 115-126. 